Int. J. Odontostomat., 9(2):255-262, 2015.

\title{
Edad de la Erupción Permanente en una Población Infantil de la Ciudad de México
}

\author{
Sequence of Permanent Eruption in an Infant Population in Mexico City
}

María del Pilar Adriano-Anaya*; Tomás Caudillo-Joya* \& Pilar Alejandra Caudillo-Adriano**

\begin{abstract}
ADRIANO-ANAYA, M. P.; CAUDILLO-JOYA, T. \& CAUDILLO-ADRIANO, P. A. Edad de la erupción permanente en una población infantil de la Ciudad de México. Int. J. Odontostomat., 9(2):255-262, 2015.

RESUMEN: Se presentan los resultados del estudio realizado en la delegación Iztapalapa en el Distrito Federal de cronología y secuencia de la erupción en población escolar y su comparación con las tablas de Hurme. El objetivo del estudio fue identificar la cronología y secuencia de la erupción en la dentición permanente de la población escolar de seis a doce años de 19 escuelas primarias públicas, ubicadas en las cuatro regiones educativas de esta delegación. Se realizó un estudio descriptivo, transversal y observacional, con una muestra por conveniencia de 5915 escolares, de 19 escuelas primarias públicas de la delegación Iztapalapa en el Distrito Federal. Se realizó la revisión de la cavidad oral tomando como diente erupcionado cuando una parte o toda la corona de éstos se encontraban erupcionadas. La información se capturó en el Programa de Cómputo SPSS versión 15.0. La edad media de erupción de los dientes permanentes de los escolares que participaron en este estudio, en el cuadrante superior fue: los incisivos centrales a los 9,3 años, incisivos laterales 9,7, los caninos 10,9, primeros premolares 10,4, segundos premolares 10,7, primeros molares 8,9 y segundos molares a los 11,2 años. En el cuadrante inferior: los incisivos centrales a los 8,9 años, incisivos laterales 9,3, caninos 10,9, primeros premolares 10,1 , segundos premolares 10,6 , primeros molares 8,8 y segundos molares a los 11,0 años. La cronología de la erupción en los escolares Iztapalapa fue más tardía con respecto a las tablas de Hurme.
\end{abstract}

PALABRAS CLAVE: cronología, emergencia, erupción, multideterminación, población escolar.

\section{INTRODUCCIÓN}

Tradicionalmente, se denomina erupción dentaria al movimiento del diente desde su posición de desarrollo dentro del proceso alveolar, hasta que alcanza una posición funcional dentro de la cavidad oral (Romero et al., 2008; Choi \& Yang, 2001) La erupción dentaria, en el sentido más estricto, dura toda la vida del diente, comprende diversas fases e implica el desarrollo embriológico de los dientes y movimientos de desplazamiento y acomodo en las arcadas. La aparición del diente en la cavidad oral recibe el nombre de emergencia o erupción dentaria (Barbería, 2001; Morón et al., 2006).

La dentición permanente consta de cuatro incisivos, dos caninos, cuatro premolares y cuatro molares en el maxilar y mandíbula, además un tercer molar que se encuentra sujeto a anomalías de número, forma y posición. Los dientes permanentes brotan con intervalos de un año entre cada grupo: el primer molar a los 6 años, los incisivos centrales maxilares y mandibulares a los 7 años, los incisivos laterales maxilares y mandibulares a los 8 años. El canino mandibular y la primera bicúspide (primer premolar) maxilar a los 9 años. El canino maxilar y la primera bicúspide (primer premolar) mandibular a los 10 años. La segunda bicúspide (segundo premolar) maxilar y mandibular a los 11 años. Los segundos molares maxilares y mandibulares a los 12 años. Los terceros molares maxilares y mandibulares de los 18 a los 30 años (Morgado Serafín \& García Herrera, 2011).

Los estudios epidemiológicos que se realicen sobre la secuencia y cronología de la erupción en la población infantil son importantes ya que nos indica las diferencias que existen con respecto a las tablas establecidas y que en ocasiones no se corresponden con la de nuestro país. Cada población y cada individuo tienen diferencias en relación a las variaciones

\footnotetext{
* Facultad de Estudios Superiores Zaragoza, Universidad Nacional Autónoma de México, México D.F., México.

** Universidad Autónoma Metropolitana-X, México D.F., México.
} 
del crecimiento y desarrollo, que están dados por factores genéticos, ambientales y del entorno sociopolítico y la erupción dental es un fenómeno de este proceso que no escapa de ello (Mayoral \& Mayoral, 1986).

Para el estudio de la cronología de erupción dentaria se han empleado diferentes tablas como la de: Hurme (1949), Moyers (1992) y Proffit \& Fields (2001), que deben servir como referencia más no como el ideal alcanzar ya que cada población tiene su especificidad determinada por el contexto donde se desarrolle, así lo indican los resultados encontrados por diferentes investigadores como son: el estudio realizado en la población infantil Vizcaína reporta que la erupción de la dentición permanente es más precoz en las niñas que en los niños. El rango de diferencia varió diente a diente, desde los cero meses para el segundo premolar maxilar, hasta los once meses para el canino mandibular, esta diferencia, además, era estadísticamente significativa para los incisivos laterales maxilares, canino y segundos molares mandibulares (Abarrategui Lopez, 2000).

En una Comunidad de Madrid España, se estudió la erupción de la dentición permanente en 752 niños y adolescentes. Los resultados encontrados son: los dientes permanentes emergen en la cavidad oral entre los 6,99 y los 12,45 años de edad en las niñas y entre los 7,06 y los 12,81 años de edad en los niños. En general, no se encontró diferencias estadísticamente significativas al momento de emergencia entre dientes homólogos (Bruna del Cojo et al., 2012).

En el Municipio de Santa Clara (Cuba), Se encontraron diferencias en un estudio en la que para incisivos, primeros molares, caninos y bicúspides, la emergencia comenzó primero en los varones, para después ser sobrepasados por las hembras que terminaron su erupción antes, con la excepción de los segundos molares que emergieron en los varones antes que en las hembras (San Miguel Pentón et al., 2011).

Investigaciones en Brasil, revelan que en los niños de 6 años el primer diente en brotar fue el incisivo central inferior para $78,7 \%$ y luego el primer molar para $75,9 \%$, en la región metropolitana de Porto Alegre (Squafk, 2008).

En el municipio de Pinar del Río en el 2012, se realizó un estudio en 209 estudiantes en el rango de edades comprendidas entre 5 y 12 años de edad de la Escuela Primaria "Pablo de la Torriente Brau" dicho estudio evidenció cambios en las fechas convencio- nales reconocidas internacionalmente, con un adelanto promedio en la erupción de un año para cada diente (Obregón et al., 2013).

En la delegación de Milpa Alta en el Distrito Federal (Caudillo Joya \& Adriano Anaya, 2012), se estudió la secuencia de la erupción en población escolar de seis a doce años, encontrando que la erupción en esta población es más retardada con respecto a las tablas de Hurme.

Otro estudio realizado en la etnia otomí en México, se observó que el $96,5 \%$ de los dientes en estos escolares erupcionan más tardíamente encontrándose diferencias que van de uno a catorce meses con respecto a lo reportado por Hurme (Taboada Aranza \& Medina García, 2005).

En Temoaya Estado de México Se examinaron 418 niños de 6 a 12 años de edad para comparar la cronología y secuencia de erupción dental de esta población con las tablas de Hurme, mostraron que la erupción de la dentición permanente es más temprana en estas y presenta mayores diferencias que las observadas entre las poblaciones mexicanas (Romo Pinales et al., 2003).

En el 2013 en niños Ugandeses se estudió el peso, talla y la erupción de los dientes permanentes, no encontrando asociación significativamente con la altura, el peso de los niños se correlacionó significativamente con la erupción en el $50 \%$ de los dientes (Kutesa et al., 2013).

La erupción dental normal se realiza en una época genéticamente establecida la cual en general corresponde con la edad cronológica. Sin embargo, es frecuente encontrar variaciones importantes en la edad en que se origina la erupción dental de un niño a otro (Moreira Díaz, 2011).

Los estudios sobre cronología de erupción dental son insuficientes en la población mexicana por lo que se hace necesario realizar este tipo de investigaciones que nos indique las edades en la que erupcionen los dientes y con ello contar con información que respondan a realidades específicas. Con el propósito de identificar las edades de la erupción en la dentición permanente de la población escolar de la delegación Iztapalapa se llevó a cabo este estudio en 19 escuelas primarias ubicadas en las cuatro regiones educativas en la que se organiza. Los resultados serán comparados con las tablas de Hurme, que han servido como referentes, buscando diferencias en la erupción permanente. 


\section{MATERIAL Y MÉTODO}

Se realizó un estudio transversal y descriptivo en una muestra por conveniencia, de $n=5915$ escolares entre las edades de seis a doce años de edad de 19 escuelas primarias, ubicadas en las cuatro regiones educativas de la delegación Iztapalapa, situada al oriente del Distrito Federal. 8 ubicadas en la Región Juárez, 2 en la Centro, 2 en San Miguel Teotongo y 7 en San Lorenzo. En estas primarias los Directores dieron la autorización para realizar el estudio.

Se consideró el diente como erupcionado cuando la corona completa o cualquier parte de ella se encontraban visibles en la cavidad oral. Para la confiabilidad de la información se estandarizaron a cinco pasantes y dos maestros obteniéndose un coeficiente de concordancia intra y inter examinador de 80 y $85 \%$. Se incluyeron en el estudio a todos los escolares que los padres o tutores otorgaron el permiso por escrito, previo consentimiento informado (Adriano Anaya \& Caudillo Joya, 2013), y se excluyeron aquellos que clínicamente al momento de la revisión presentaron algún problema de salud. La revisión de la cavidad oral, se llevó a cabo en aulas de clase otorgadas en cada una de las primarias, utilizando espejos

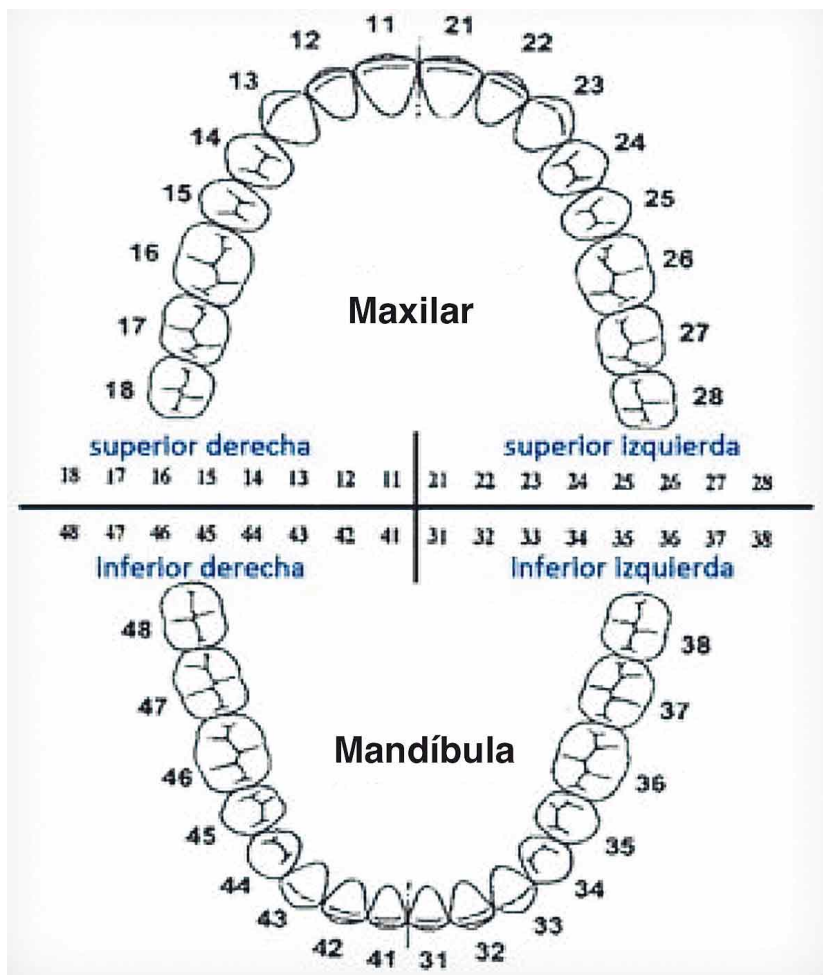

Fig. 1. Sistema de numeración dental FDI. dentales, sonda WHO y luz natural, aplicando las barreras de protección planteadas por la Norma Oficial Mexicana para el control y manejo de infecciones orales, Norma Oficial Mexicana NOM-087-ECOL-SSA12002 (2002). Se marcó en el instrumento de recolección el nombre del escolar, edad, sexo, en el apartado del odontograma con el código 1 cuando el diente se encontraba erupcionado y 2 no se hallaba presente en la cavidad oral. Los dientes extraídos se tomaron como erupcionados. La nomenclatura utilizada para identificar a los dientes permanentes fue la propuesta por la FDI (Fig. 1).

La información se capturó en el Programa de Cómputo SPSS versión 15.0 donde se obtuvieron:

- Número de escolares participantes por cada una de las edades.

- Porcentaje de erupción de los dientes de los escolares por edad.

Se cambió la variable erupcionado en la base de datos por las edades, se tomó el promedio con las edades del diente erupcionado.

Se obtuvo la t de Student para una muestra, comparando la media muestral de los escolares de Iztapalapa contra la media del grupo normativo (Hurme), se obtuvo la diferencia significativa de cada diente. Así mismo se categorizó por sexo, realizando el mismo procedimiento.

\section{RESULTADOS}

En este estudio participaron 5915 escolares entre seis a doce años de 19 primarias de las cuatro regiones educativas de la delegación Iztapalapa, localizada en el Oriente del Distrito Federal (Tabla I). Del total de la muestra, 932 escolares fueron de seis años, 992 de siete, 945 de ocho, 981 de nueve, 1022 de diez, 795 de once y 248 escolares de doce años. El $50,1 \%$ fueron del sexo femenino y el $49,9 \%$ del masculino.

Al momento de la revisión, 335 escolares de seis años, y 25 de siete no tuvieron ningún diente permanente erupcionado.

Los dientes que se encontraron erupcionados en un $100 \%$ en esta población a los doce años fueron: los primeros molares maxilares y mandibulares, inci- 
Tabla I. Distribución de los escolares por primarias.

\begin{tabular}{lccc}
\hline Primaria & Frecuencia & $\%$ & Regiones Educativas \\
\hline 1. Alicia Martínez Montoya & 365 & 6,2 & Juárez \\
2. José Palomares Quiroz & 470 & 7,9 & Centro \\
3. Luis Pasteur & 354 & 6,0 & Juárez \\
4. Antonio Castro Leal & 363 & 6,1 & Juárez \\
5. Ignacio Chávez Matutino & 192 & 3,2 & Juárez \\
6. Ignacio Chávez Vespertino & 407 & 6,9 & Juárez \\
7. Julio Cortázar Matutino & 124 & 2,1 & Juárez \\
8. Julio Cortázar Vespertino & 83 & 1,4 & Juárez \\
9. Miguel de Cervantes Saavedra & 180 & 3,0 & Centro \\
10. Emperador Izcoatl & 205 & 3,5 & Juárez \\
11. Mitla & 312 & 5,3 & San Lorenzo \\
12. Reforma Agraria & 249 & 4,2 & San Lorenzo \\
13. Juan de Mata Rivera & 511 & 8,6 & San Lorenzo \\
14. Miguel Sánchez & 350 & 5,9 & San Lorenzo \\
15. Plan de San Luis & 248 & 4,2 & San Lorenzo \\
16. Fray Bartolomé de las Casas & 407 & 6,9 & San Lorenzo \\
17. Estado de Colima & 310 & 5,2 & San Lorenzo \\
18. Hermanos Serdán & 409 & 6,9 & San Miguel Teotongo \\
19. Mariano Hidalgo & 376 & 6,4 & San Miguel Teotongo \\
Total & 5915 & 100,0 & \\
\hline
\end{tabular}

sivos centrales y laterales maxilares y mandibulares y el primer premolar mandibular izquierdo (Tabla II).

Con respecto a la t para muestras independiente se encontró significancia estadística, en el primer cuadrante en el diente $14,13,12$, y 11 erupcionando primero estos dientes en el sexo femenino (Tabla III). En el segundo cuadrante existen diferencias en los órganos dentarios $21,22,23,25$ y 27 erupcionaron primero estos dientes en el sexo femenino (Tabla III). En el tercer cuadrante hay diferencias en el 37,35 y 33 , estos dientes erupcionaron primero en las niñas (Tabla III). En el cuadrante inferior derecho hay diferencias en el 43 y 44, estos dientes erupcionaron primero en el sexo femenino (Tabla III).

La edad media en la que erupcionaron los dientes permanentes de los escolares que participaron en este estudio, en el cuadrante superior fue: los incisivos centrales a los 9,3 años, incisivos laterales 9,7, los caninos 10,9 , primeros premolares 10,4 , segundos premolares 10,7 , primeros molares 8,9 y segundos molares a los 11,2 años (Tabla IV).

La edad media en la que erupcionaron los dientes permanentes de los escolares que participaron en este estudio, en el cuadrante inferior fue: los incisivos centrales a los 8,9 años, incisivos laterales 9,3 , caninos 10,9 , primeros premolares 10,1 , segundos premolares 10,6 , primeros molares 8,8 y segundos molares a los 11,0 años (Tabla IV).

Al comparar la erupción de los escolares de Iztapalapa y los estudios de Hurme, en el cuadrante superior, los caninos en ambos sexos erupcionaron primero en Iztapalapa, así como los segundos premolares. El resto de los dientes erupcionaron primero en los escolares del estudio de Hurme (Tabla V). Con respecto a la prueba t para una muestra, se encontraron diferencias estadísticas entre la edad de la erupción de los dientes permanentes por sexo de los escolares de Iztapalapa con las tablas de Hurme.

En el cuadrante inferior los únicos dientes que erupcionaron primero en los escolares de Iztapalapa fueron los segundos premolares, el resto de los dientes lo hicieron primero en el estudio de Hurme.

\section{DISCUSIÓN}

El proceso de la erupción dentaria hasta el momento actual ha sido estudiada por fenómenos que intervienen en este de manera aislada, separada, no reconociendo otros, en donde se observa la articulación entre ellos, es importante el reconocer este proceso como complejo y en consecuencia comprender- 
ADRIANO-ANAYA, M. P.; CAUDILLO-JOYA, T. \& CAUDILLO-ADRIANO, P. A. Edad de la erupción permanente en una población infantil de la Ciudad de México. Int. J. Odontostomat., 9(2):255-262, 2015.

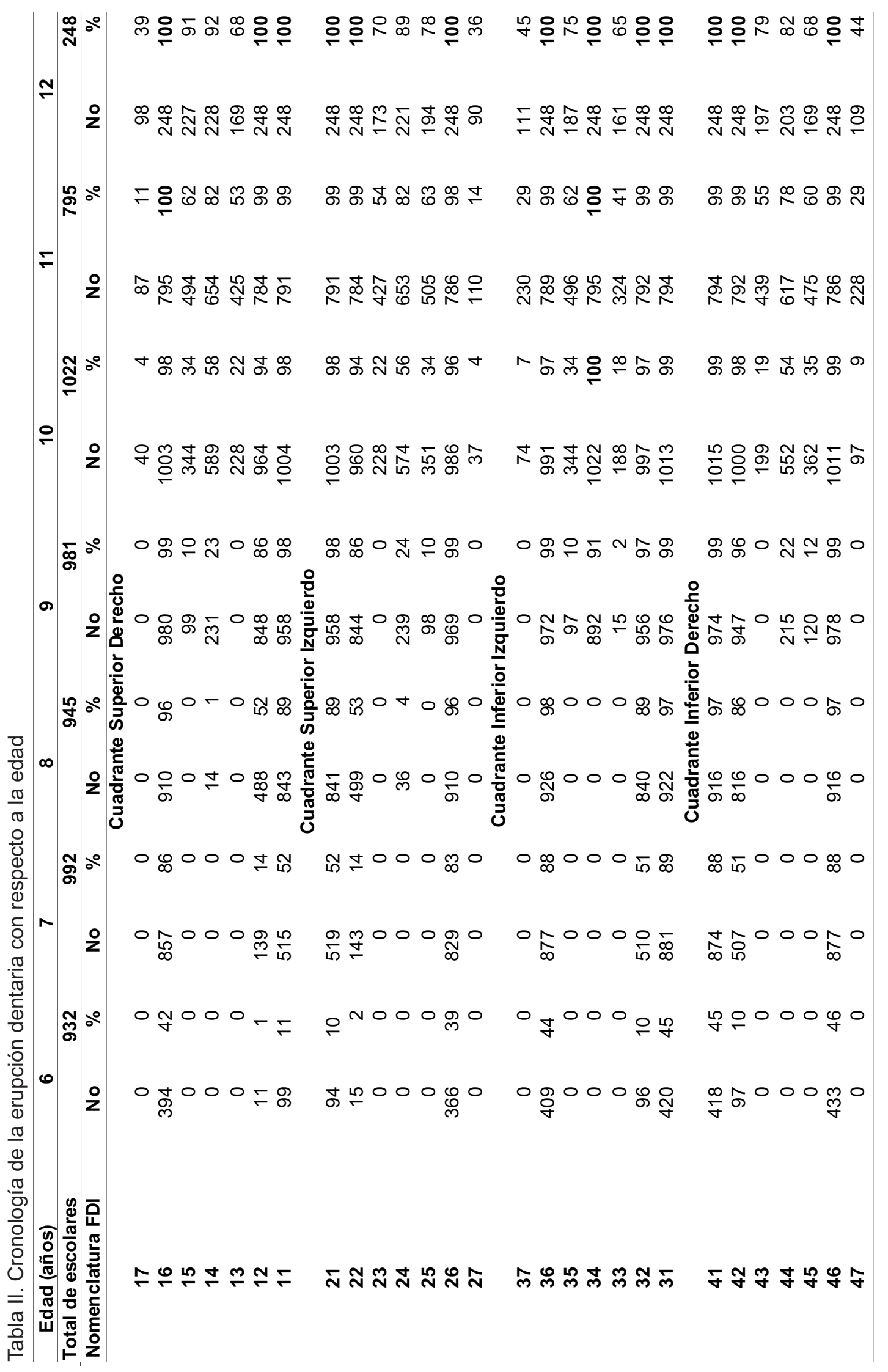


Tabla III. Diferencias estadísticas de la cronología de la erupción por sexo.

\begin{tabular}{|c|c|c|c|}
\hline $\begin{array}{c}\text { Nomenclatura } \\
\text { FDl }\end{array}$ & Significancia & $\begin{array}{c}\text { Edad } \\
\text { Masculino }\end{array}$ & $\begin{array}{c}\text { Edad } \\
\text { Femenino }\end{array}$ \\
\hline \multicolumn{4}{|c|}{ Primer cuadrante (superior derecho) } \\
\hline 17 & 0,102 & 11,4 & 11,3 \\
\hline 16 & 0,121 & 8,11 & 8,10 \\
\hline 15 & 0,112 & 10,9 & 10,8 \\
\hline 14 & $0,050^{*}$ & 10,6 & 10,5 \\
\hline 13 & $0,004^{*}$ & 11,1 & 10,10 \\
\hline 12 & $0,000^{*}$ & 9,9 & 9,7 \\
\hline 11 & $0,010^{*}$ & 9,4 & 9,2 \\
\hline \multicolumn{4}{|c|}{ Segundo cuadrante (superior izquierdo) } \\
\hline 21 & $0,003^{*}$ & 9,4 & 9,2 \\
\hline 22 & $0,000^{*}$ & 9,9 & 9,7 \\
\hline 23 & $0,009^{*}$ & 11,0 & 10,10 \\
\hline 24 & 0,115 & 10,6 & 10,5 \\
\hline 25 & $0,021^{*}$ & 10,9 & 10,8 \\
\hline 26 & 0,301 & 8,11 & 8,10 \\
\hline 27 & $0,038^{*}$ & 11,4 & 11,2 \\
\hline \multicolumn{4}{|c|}{ Tercer cuadrante (Inferior izquierdo) } \\
\hline 37 & $0,020^{*}$ & 11,3 & 11,1 \\
\hline 36 & 0,080 & 8,11 & 8,10 \\
\hline 35 & $0,009^{*}$ & 10,9 & 10,7 \\
\hline 34 & 0,744 & 10,1 & 10,2 \\
\hline 33 & $0,019^{*}$ & 11,00 & 10,10 \\
\hline 32 & 0,108 & 9,4 & 9,2 \\
\hline 31 & 0,088 & 8,11 & 8,10 \\
\hline \multicolumn{4}{|c|}{ Cuarto cuadrante (inferior derecho) } \\
\hline 41 & 0,063 & 8,11 & 8,10 \\
\hline 42 & 0,060 & 9,4 & 9,3 \\
\hline 43 & $0,001^{*}$ & 11,1 & 10,11 \\
\hline 44 & $0,024^{*}$ & 10,7 & 10,5 \\
\hline 45 & 0,073 & 10,8 & 10,7 \\
\hline 46 & 0,098 & 8,10 & 8,10 \\
\hline 47 & 0,174 & 11,1 & 10,11 \\
\hline
\end{tabular}

*Dientes en los que hay significancia estadística, con respecto a la t.

Tabla IV. Edad media de la erupción permanente en los escolares.

\begin{tabular}{lrlr}
\hline \multicolumn{1}{c}{ Cuadrante superior } & \multicolumn{2}{c}{ Cuadrante inferior } \\
\hline Incisivos centrales & 9,3 & Incisivos centrales & 8,9 \\
Incisivos laterales & 9,7 & Incisivos laterales & 9,3 \\
Caninos & 10,9 & Caninos & 10,9 \\
Primeros premolares & 10,4 & Primeros premolares & 10,1 \\
Segundos premolares & 10,7 & Segundos & 10,6 \\
Primeros molares & 8,9 & Primeros molares & 8,8 \\
Segundos molares & 11,2 & Segundos molares & 11,0 \\
\hline
\end{tabular}

lo en su multidimensionalidad (Adriano Anaya \& Caudillo Joya), en donde interactúan factores ambientales, traumáticos, hereditarios y genéticos, pero, también intervienen otros más generales, tales como los étnicos, sociales, económicos, culturales entre otros. Por lo que es necesario realizar éste tipo de estudios en contextos específicos para identificar las edades de erupción y tomar las tablas de: Hurme, Moyers y Proffit \& Fields como parámetros de comparación útiles ya que estas fueron realizadas en contextos muy particulares y tiene su aplicación para dicha realidad, es por ello que en México se deben realizar estudios sobre este proceso con el fin de contar con sus propias tablas de cronología y secuencia de la erupción.

Una de las limitaciones de este estudio es que no se realizó asociación con otro tipo de variables como puede ser la obesidad o la desnutrición.

En términos generales la edad de erupción de los escolares de Iztapalapa y los estudios de Hurme, en el cuadrante superior, los caninos y los segundos premolares en ambos sexos erupcionaron primero en Iztapalapa. El resto de los dientes lo hicieron de acuerdo a la tabla de erupción realizada por Hurme. En el cuadrante inferior los únicos dientes que erupcionaron primero en los escolares de Iztapalapa fueron los segundos premolares, el resto de los dientes lo hicieron de acuerdo al estudio de Hurme. Con respecto al sexo todos los dientes erupcionaron primero en el sexo femenino que en el masculino, similar al estudio realizado en la población infantil de Vizcaína y en una Comunidad de Madrid España (Bruna del Cojo et al.).

No así en el estudio de Santa Clara Cuba (San Miguel Pentón et al.), donde la erupción dentaria se realizó primero en los varones.

Los resultados de la cronología de la erupción dentaria de los escolares de la Delegación de Iztapalapa fueron similares al estudio realizado en la delegación Milpa Alta (Adriano Anaya \& Caudillo Joya).

De los 5915 escolares que participaron en este estudio, a los seis años el $46 \%$ de ellos tenían erupcionado el primer molar mandibular derecho, posteriormente el mandibular izquierdo (44\%), el maxilar derecho (42\%) y finalmente el maxilar izquierdo (42\%). No así en el estudio de Brasil, en la región metropolitana de Porto Alegre, que a los 6 años, el primer diente 
Tabla V. Edad media de la erupción por sexo de los escolares de Iztapalapa con las de Hurme

\begin{tabular}{|c|c|c|c|c|}
\hline \multicolumn{5}{|c|}{ Cuadrante Superior } \\
\hline \multirow{2}{*}{ Dientes } & \multicolumn{2}{|c|}{ Iztapalapa } & \multicolumn{2}{|c|}{ Hurme } \\
\hline & $\mathbf{F}$ & $\mathbf{M}$ & $\mathbf{F}$ & $\mathbf{M}$ \\
\hline Centrales & $9,20 \pm 2,5$ & $9,32 \pm 2,6$ & 7,20 & 7,47 \\
\hline Laterales & $9,61 \pm 2,9$ & $9,82 \pm 2,9$ & 8,20 & 8,67 \\
\hline Caninos & $10,87^{*} \pm 2,2$ & $11,01^{*} \pm 1,9$ & 10,98 & 11,69 \\
\hline Primeros Premolares & $10,46 \pm 2,7$ & $10,54 \pm 2,6$ & 10,03 & 10,40 \\
\hline Segundos Premolares & $10,64^{*} \pm 2,4$ & $10,72 * \pm 2,3$ & 10,88 & 11,18 \\
\hline Primeros Molares & $8,87 \pm 2,0$ & $8,94 \pm 2,0$ & 6,22 & 6,40 \\
\hline \multirow[t]{2}{*}{ Segundos Molares } & $\begin{array}{r}11,19 \pm 1,2 \\
t=d f=2\end{array}$ & $\begin{array}{l}11,35 \pm 1,1 \\
p=0,000\end{array}$ & 12,27 & 12,68 \\
\hline & \multicolumn{2}{|c|}{ Cuadrante Inferior } & & \\
\hline Centrales & $8,8 \pm 1,8$ & $8,9 \pm 1,9$ & 6,26 & 6,54 \\
\hline Laterales & $9,2 \pm 2,5$ & $9,3 \pm 2,6$ & 7,34 & 7,70 \\
\hline Caninos & $10,9 \pm 2,0$ & $11,0 \pm 1,8$ & 9,86 & 10,79 \\
\hline Primeros Premolares & $10,14 \pm 2,4$ & $10,13 \pm 2,5$ & 10,18 & 10,82 \\
\hline Segundos Premolares & $10,63^{*} \pm 2,4$ & $10,76^{*} \pm 2,2$ & 10,89 & 11,47 \\
\hline Primeros Molares & $8,8 \pm 1,9$ & $8,9 \pm 1,9$ & 5,94 & 6,21 \\
\hline \multirow[t]{2}{*}{ Segundos Molares } & $11,02 \pm 1,6$ & $11,17 \pm 1,4$ & 11,66 & 12,12 \\
\hline & $t=d f=2$ & $p=0,000$ & & \\
\hline
\end{tabular}

en brotar fue el incisivo central mandibular $(78,7 \%)$ y luego el primer molar $(75,9 \%)$.

El $100 \%$ de dientes erupcionados a los doce años fueron: 16, 12, 11, 21, 22, 26, 36, 34, 32, 31, 41, 42, 46. La edad promedio de los primeros dientes permanentes en erupcionar fueron los primeros molares mandibulares a los 8,8 años.
El tener estudios sobre la edad en que erupcionan los dientes permanentes en contextos específicos como es el de los escolares de Iztapalapa es muy importante para realizar Diagnósticos, Planes de tratamientos y diferentes programas de intervención ya sean preventivos, curativos o de rehabilitación en donde prevalezca el propósito de que los escolares gocen de una buena salud oral.

ADRIANO-ANAYA, M. P.; CAUDILLO-JOYA, T. \& CAUDILLO-ADRIANO, P. A. Age of permanent eruption in an infant population in Mexico City. Int. J. Odontostomat., 9(2):255-262, 2015.

ABSTRACT: Chronology and eruption sequence study results in the Iztapalapa delegation from México City are presented in comparison with the Hurme tables. The objective of this study was to identify the chronology and sequence of the permanent teeth eruption in a school population between six and twelve years old from 19 public elementary schools, located in the four educational regions in the Iztapalapa delegation, Mexico City. We carried out a cross-sectional, observational and descriptive study, with a convenience sample of 5915 students from 19 elementary public schools in the Iztapalapa delegation, Mexico City. The collection of information was performed from September 2012 to November 2013, the oral exam was done considering as an erupted tooth, when a part or the entire crown were found in the mouth. The information was collected in a special poll designed for this study, in this poll we recorded with the number one, if the tooth was erupted and with the number two if it was not found in the mouth. This information was captured in the SPSS software versión 15.0. When comparing the eruption from the students in Iztapalapa and the Hurme studies, in the upper quadrant, canines as well as the second premolars in both sexes erupted first in Iztapalapa. The rest of the teeth erupted first in the students from the Hurme studies. In the lower quadrant the only teeth that erupted first were second premolars, the rest of the teeth erupted first in the Hurme studies. The chronology of the eruption in Iztapalapa school was later regarding Hurme tables.

KEY WORDS: cronology, emergency, eruption, multidetermination, school population. 


\section{REFERENCIAS BIBLIOGRÁFICAS}

Abarrategui Lopez, I.; Gorritxo Gil, B. \& Goiriena de Gandarias, F. J. Edades medias de erupción para la dentición permanente. Rev. Esp. Ortod., 30:23-9, 2000.

Adriano Anaya, M. P. \& Caudillo Joya, T. Valores éticos en odontología. Odontol. Actual,10(124):58-60, 2013.

Barbería, L. E. Erupción dentaria. Prevención y tratamiento de sus alteraciones. Pediatr. Integral, 6(3):229-40, 2001.

Bruna del Cojo, M.; Gallardo López, N. E.; Mourelle Martínez, M. R. \& De Nova García, M. J. Estudio de la erupción de la dentición permanente en niños y adolescentes de la Comunidad de Madrid (España). Cient. Dent., 9(3):15964, 2012.

Caudillo Joya, T. \& Adriano Anaya, M. P. Cronología de la erupción de dientes permanente en escolares de seis a doce años. Odontol. Pediatr., 1(2):44-8, 2012.

Choi, N. K. \& Yang, K. H. A study on the eruption timing of primary teeth in Korean children. A. S. D. C. J. Dent. Child, 68(4):244-9, 2001.

Hurme, V. O. Ranges of normalcy in the eruption of permanent teeth. J. Dent. Child, 16(2):11-5, 1949.

Kutesa, A.; Nkamba, E. M.; Muwazi, L.; Buwembo, W. \& Rwenyonyi, C. M. Weight, height and eruption times of permanent teeth of children aged 4-15 years in Kampala, Uganda. B. M. C. Oral Health, 13:15, 2013.

Mayoral, J. \& Mayoral, G. Ortodoncia. Principios fundamentales y prácticos. $4^{\mathrm{a}}$ ed. La Habana, Editorial CientíficoTécnica, 1986.

Moreira Díaz, E. E. Erupción dental. La secuencia de erupción. Las tres preguntas más frecuentes. Dentista en la Red, 2011. Disponible en: http://dentistaenlared.com/ ortodoncia_erupcion\%20dental.html.

Morgado Serafín, D. \& García Herrera, A. Cronología y variabilidad de la erupción dentaria. Policlínico docente Dr. Mario Páez Inchausti. Medeciego, 17(Supl. 2), 2011. Disponible en: http://www.bvs.sld.cu/revistas/mciego/ vol17_supl2_\%202011/pdf/T16.pdf

Morón B., A.; Santana, Y.; Pirona, M.; Rivera, L.; Rincón, M. C. \& Pirela, A. Cronología y secuencia de erupción de dientes permanentes en escolares wayúu. Parroquia Idelfonso Vásquez. Municipio Maracaibo-estado Zulia. Acta Odontol. Venez., 44(1), 2006.

Moyers, R. E. Manual de ortodoncia para el estudiante y el odontólogo general. Buenos Aires, Editorial Mundi, 1992.
Norma Oficial Mexicana NOM-087-ECOL-SSA1-2002, Protección ambiental - Salud ambiental - Residuos peligrosos biológico-infecciosos-Clasificación y especificaciones de manejo. México D. F., Diario Oficial de la Federación, Estados Unidos Mexicanos, 2002. Disponible en: http://www.salud.gob.mx/unidades/cdi/nom/ 087ecolssa.html.

Obregón, T. C.; Sosa Hernández, H. P.; Matos Rodríguez, A. \& Díaz Pacheco, C. Orden y cronología de brote en dentición permanente. Rev. Cienc. Méd., 17(3):112-22, 2013.

Proffit, W. R. \& Fields, H. Ortodoncia contemporánea. Teoría y práctica. Washington, Ediciones Harcout, 2001.

Romero R., M. A.; Chávez, E. \& Barrero, J. Prevalencia y secuencia de erupción en el maxilar inferior en pacientes escogidos del Diplomado de Ortodoncia Interceptiva U.G.M.A. Rev. Latinoam. Ortod. Odontopediatria, 2008. Disponible en: http://www.ortodoncia.ws/publicaciones/ 2008/art10.asp

Romo Pinales, M. R.; Hernández Zavala, M. S.; De Jesús Herrera, M. I. \& Rubio Cisneros, J. Perfiles de erupción dental en población escolar en un Municipio del Estado de México. Bol. Med. Hosp. Infant. Mex., 60(5):499-515, 2003.

San Miguel Pentón, A.; Veliz Concepción, O. L.; Escudero Alemán, R. Z.; Calcines Ferrer, M. E. \& Ortega Romero, L. Cronología de emergencia de la dentición permanente en niños del municipio de Santa Clara: Parte I. Rev. Cuba. Estomatol., 48(3):11-22, 2011.

Squafk, K. Presença dentes permanentes erompidos em escolares da regiõao metropolitana de Porto Alegre. Stomatos., 14(26):11-8, 2008.

Taboada Aranza, O. \& Medina García, J. L. Cronología de erupción dentaria en escolares de una población indígena del Estado de México. Rev. A. D. M., 62(3):94-100, 2005.

Dirección para Correspondencia:

María del Pilar Adriano Anaya

Facultad de Estudios Superiores Zaragoza

Universidad Nacional Autónoma de México

Av. Guelatao 66, Iztapalapa

Ejército de Oriente, 09230

Ciudad de México, D.F.

MÉXICO

Email: adriano124@hotmail.com 